\title{
Gestação gemelar de mola hidatiforme completa com feto vivo
}

\section{Twin pregnancy with complete hydatidiform mole and living fetus}

Daniela Angerame Yela'; Anderson Pinheiro²; João Paulo Leonardo Pinto²; Liliana Andrade ${ }^{3}$

\begin{tabular}{l|l}
\multicolumn{1}{c|}{ Unitermos } & resumo \\
$\begin{array}{l}\text { Gestação gemelar } \\
\text { Mola hidatiforme completa }\end{array}$ & $\begin{array}{l}\text { A gestação gemelar de uma mola hidatiforme completa coexistindo com um feto vivo é uma entidade } \\
\text { rara. Os poucos casos descritos na literatura mostram que, em geral, esse tipo de gestação cursa com } \\
\text { risco aumentado de aborto espontâneo, parto prematuro, morte fetal intraútero, sangramento, pré- } \\
\text { Feto vivo }\end{array}$ \\
$\begin{array}{l}\text { eclâmpsia e doença trofoblástica persistente. Neste artigo, descreveremos o caso de uma primigesta } \\
\text { de } 20 \text { anos de idade que apresentou uma gestação gemelar de feto vivo com mola completa, a qual } \\
\text { transcorreu sem intercorrências e complicações e cujo diagnóstico diferencial com degeneração da } \\
\text { placenta foi feito com anatomopatológico. }\end{array}$
\end{tabular}

abstract

Twin pregnancy with a complete hydatidiform mole and a living fetus is a rare entity. According to the few cases described in the literature, this kind of gestation commonly has higher risks of miscarriage, preterm delivery, intrauterine fetal death, bleeding, preeclampsia and persistent trophoblastic disease. This study reports the case of a 20 years old primiparous patient with complete hydatidiform mole and living fetus, whose pregnancy was successfully carried to term. The differential diagnosis with placental degeneration was reached through anatomopathological investigation.

\section{key words}

Twin pregnancy

Complete hydatidiform

mole

Live fetus

1. Doutora em Tocoginecologia; médica contratada da Universidade Estadual de Campinas (UNICAMP).

2. Médico residente da UNICAMP; pós-graduando em Tocoginecologia.

3. Doutora em Tocoginecologia; docente da UNICAMP. 


\section{Introdução}

A gestação gemelar de uma mola hidatiforme completa coexistindo com um feto vivo é uma entidade rara. Sua incidência varia de uma em 22 mil até uma em 100 mil

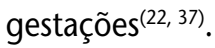

Quando se observa uma placenta com uma degeneração molar e um feto vivo, temos de considerar as seguintes hipóteses: mola parcial com feto triploide e gestação gemelar com feto normal e mola completa (GGMC) ${ }^{(9)}$. A GGMC aumenta o risco de aborto espontâneo, parto prematuro, morte fetal intraútero, sangramento, pré-eclâmpsia, doença trofoblástica persistente e alguns casos que terminam em parto com feto vivo ${ }^{(9,36)}$.

Neste artigo será descrito inicialmente um caso de gestação gemelar coexistindo com mola hidatiforme e feto normal. A seguir, será apresentada a revisão da literatura.

\section{Relato do caso}

Primigesta, 20 anos de idade, branca, gestante de 17 semanas, com subunidade beta da gonadotrofina coriônica humana ( $\beta \mathrm{HCG}$ ) inicial de $178.510 \mathrm{mUl} / \mathrm{mol}$. A ecografia mostrou um feto vivo com biometria fetal compatível com 16/17 semanas de gestação, batimentos cardíacos e movimentos fetais presentes. A borda lateral da placenta apresentava região heterogênea, com áreas císticas de permeio medindo $126 \times 80 \mathrm{~mm}$ e com plano de clivagem em relação à placenta normal. A imagem ultrassonográfica inicial é mostrada na Figura 1.

O diagnóstico ultrassonográfico foi de gestação gemelar com feto de 16/17 semanas e mola hidatiforme completa, isso porque havia um plano de clivagem separando a pla-

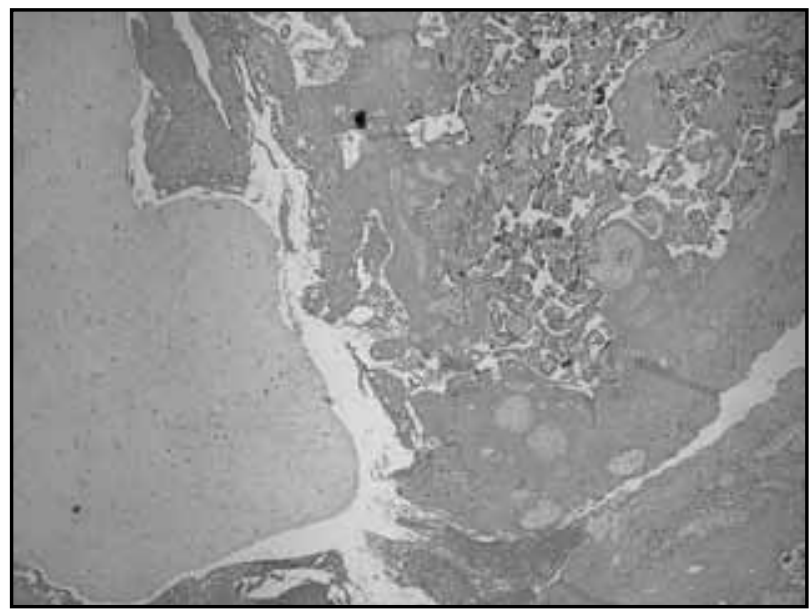

Figura 1 - Imagem ultrassonográfica inicial centa normal da mola e, assim, descartou-se degeneração hidrópica da placenta; como o feto era normal, descartou-se mola parcial.

A paciente iniciou acompanhamento no pré-natal especializado no Centro de Atenção Integral à Saúde da Mulher da Universidade Estadual de Campinas (CAISM/ UNICAMP). Para excluir o diagnóstico de mola parcial, foi realizada aminiocentese para coleta de cariótipo. Não houve crescimento celular e o exame foi repetido sem sucesso.

Optado pelo seguimento da gestação, que ocorreu sem intercorrências. $\mathrm{O}$ acompanhamento ultrassonográfico mostrava crescimento fetal adequado e áreas císticas à borda placentária sem invasão miometrial. A evolução do $\beta$ HCG durante a gestação é mostrada na Tabela 1.

\section{Tabela 1 Evolução do $\beta$ HCG duranta a gestação.}

$\begin{array}{cc}\text { Semanas de gestação } & \beta H C G(\mathrm{mUl} / \mathrm{mol}) \\ 16 / 17 & 163.400 \\ 20 / 21 & 141.700 \\ 23 / 24 & 155.100 \\ 28 / 29 & 192.500 \\ 34 / 35 & 860.000\end{array}$

$\beta H C G$ : subunidade beta da gonadotrofina coriônica humana.

O parto se deu às 40 semanas, do tipo cesárea, devido à distócia funcional. O recém-nascido de termo nasceu com $3.100 \mathrm{~g}, 49 \mathrm{~cm}$, Apgar 9/10, do sexo feminino e com uma placenta de $870 \mathrm{~g}$ com dequitação completa seguida de eliminação de vesículas.

No anatomopatológico, a macroscopia descrevia uma placenta de $870 \mathrm{~g}$ medindo $14 \times 16 \times 4 \mathrm{~cm}$, cuja face materna mostrava cotilédones íntegros e face fetal lisa, violácea com vasos túrgidos e membranas lisas e translúcidas e outra massa medindo $9 \times 14 \times 4 \mathrm{~cm}$ com vesículas de $1 \mathrm{~cm}$, conteúdo hialino, pediculadas em toda a extensão confirmando o diagnóstico de mola completa associada a uma placenta normal de terceiro trimestre de gestação. Imagem macroscópica e microscópica da placenta é mostrada nas Figuras 2 e $\mathbf{3}$.

O $\beta$ HCG pós-parto foi de $13.580 \mathrm{mUl} / \mathrm{mol}$ e após seis dias, $987 \mathrm{mUl} / \mathrm{mol}$. Após 45 dias, o $\beta \mathrm{HCG}$ encontrava-se negativo. $\mathrm{O}$ método anticoncepcional utilizado no pósparto foi a Depoprovera ${ }^{\circledR}$.

O cariótipo do recém-nascido foi 46XX e a criança encontra-se hoje com 2 anos de idade e com boa saúde. 


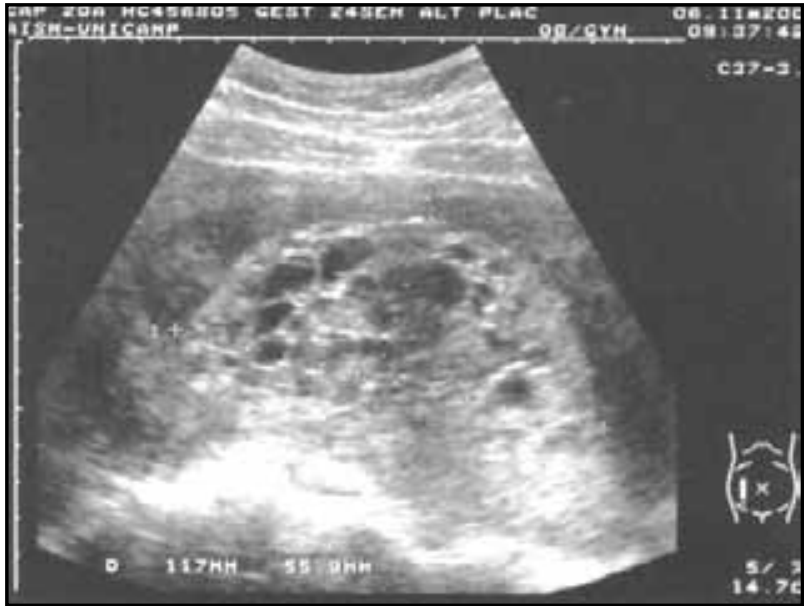

Figura 2 - Imagem macroscópica da placenta

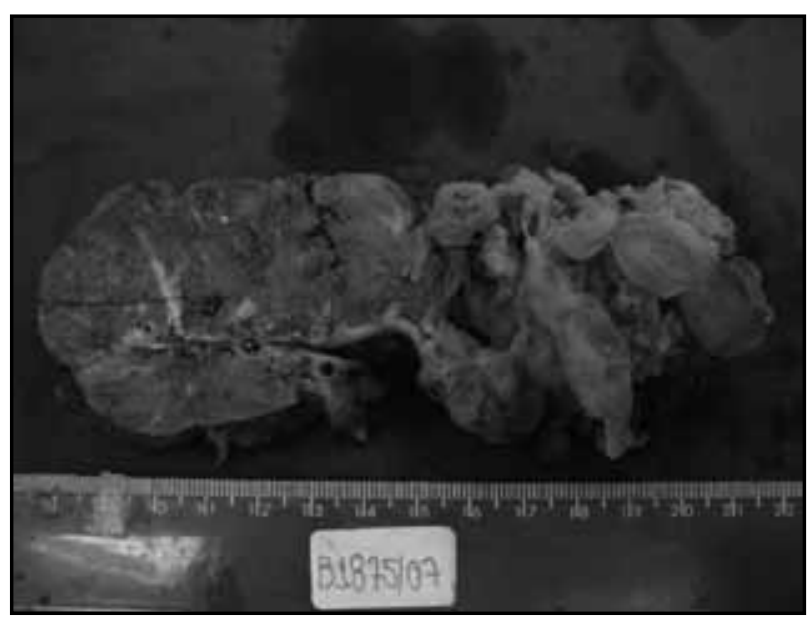

Figura 3 - Imagem microscópica da placenta

\section{Discussão}

A mola hidatiforme completa apresenta um cariótipo 46XX fruto de fertilização de um óvulo cego com um espermatozoide haploide, que tem seu conteúdo duplicado (origem paterna). Apresenta risco de $12 \%$ a $20 \%$ de desenvolver a doença trofoblástica gestacional persistente (TGP). Esse risco é mais alto se comparado ao apresentado pela mola parcial (4\%), que tem tem cariótipo triploid, resultado da fertilização de um óvulo haploide por dois espermatozoides ${ }^{(6,34)}$.

A incidência de mola hidatiforme completa é de aproximadamente uma para mil gestações, enquanto a da mola parcial é de três para 100 gestações $^{(31)}$.

Quando encontramos um feto vivo com uma placenta com degeneração molar, devemos questionar duas possibilidades: ser uma mola parcial ou uma gestação gemelar de mola completa e feto vivo.

O nível de $\beta$ HCG é usado como marcador em gestações molares, no entanto ele perde valor quando na presença de gestação gemelar molar com feto viável, uma vez que os níveis podem estar normais ${ }^{(37)}$. Alguns autores descrevem como sendo níveis de $\beta$ HCG maiores, principalmente no primeiro trimestre ${ }^{(30)}$.

Quando há suspeita ecográfica de GGMC, o diagnóstico deve ser feito por métodos invasivos com a determinação do cariótipo do feto, que ainda pode trazer dúvidas porque, apesar de raro, a presença de cariótipo normal em gestação molar parcial pode ser encontrada. O método de certeza é por elaboração citogenética, que identifica o cariótipo como sendo exclusivamente paterno ${ }^{(37)}$. Quando não disponível, o diagnóstico final é realizado com a análise histopatológica da placenta após o termino da gestação(30). Recentemente, no Japão, foram descritos três casos de GGMC diagnosticados pela análise do DNA placentário. Outro estudo japonês relatou que os métodos tradicionais para detecção da GGMC são inadequados e que o ideal para seu diagnóstico é a análise do $\mathrm{DNA}^{(17,29,35)}$.

O manuseio da GGMC é difícil, uma vez que pode ser seguida de diversas complicações, entre elas, sangramento vaginal, hipertireoidismo, hiperemese gravídica, parto prétermo, pré-eclâmpsia severa (complicação mais comum [30\%]), cisto teca-luteínico (23\%), doença tromboembólica com acometimento pulmonar, TGP em $30 \%$ dos casos e metástases (22\%). Essas complicações, principalmente as relacionadas com a característica tumoral da doença, colocam em discussão a viabilidade de se manter a gestação ou de interrompê-la assim que é realizado o diagnóstico ${ }^{(11,22,28,30,31,37)}$. Entretanto, alguns estudos mostram que $40 \%$ dessas gestações resultam em feto viável e normal(20, 32).

No caso descrito, houve dúvida de diagnóstico no momento do primeiro exame ultrassonográfico. Mesmo assim optou-se por aguardar a evolução da gestação e realizar cariotipagem e ecografia seriada. Como a paciente foi evoluindo sem complicações e o desenvolvimento fetal estava adequado, a hipótese que permanecia foi mesmo a de gestação com degeneração molar placentária. O caso só teve seu diagnóstico confirmado de gestação gemelar com um feto vivo e outro fruto de mola completa após o parto e com o exame histopatológico da placenta.

Recentemente, na Coreia, um estudo que avaliou casos de doença trofoblástica entre 1998 e 2008 encontrou seis casos de GGMC, sendo que apenas um evoluiu com gestação com feto vivo; os demais tiveram a gestação interrompida antes de 21 semanas, devido às complicações maternas ${ }^{(19)}$.

Em uma revisão de literatura com 29 trabalhos, encontram-se descritos 159 casos de GGMC, sendo que, 
destes, apenas $56(35 \%)$ apresentaram término da gestação com feto vivo. Grande parte dessas gestações apresentou complicações, como pré-eclâmpsia e doença trofoblástica persistente. Esses dados são demonstrados na Tabela 2.

A ocorrência de feto vivo na GGMC é estimada na literatura entre $29 \%$ e $38 \%$, enquanto de pré-eclâmpsia e TGP é de 30\%. O risco de parto pré-termo é estimado em
$62 \%{ }^{(11)}$. Praticamente não há correlação com a presença de cistos teca-luteínicos ${ }^{(23)}$. Morte fetal intrauterina espontânea é muito comum, principalmente antes de 24 semanas de idade gestacional ${ }^{(23)}$.

Quando comparamos este caso com os apresentados na literatura, podemos dizer que ele é raro, uma vez que a gestação terminou em feto vivo, apresentando ausência de complicações maternas e fetais.

\section{Tabela 2 Descrição de 159 casos de GGMC}

\begin{tabular}{|c|c|c|c|c|c|c|}
\hline Estudo & $\mathrm{N}$ & $\begin{array}{c}\text { Idade } \\
\text { gestacional }\end{array}$ & $n$ & Feto vivo & Pré-eclâmpsia & TGP \\
\hline Shahabi et al. ${ }^{(33)}$ & 2 & 39 & 1 & 1 & - & 1 \\
\hline Block; Merrill(4) & 2 & $35 / 36$ & 0 & 2 & - & 1 \\
\hline Miller et al. ${ }^{(25)}$ & 4 & 38 & 3 & 1 & - & 3 \\
\hline Steller et al. ${ }^{(34)}$ & 8 & 31 & 7 & 1 & 2 & 5 \\
\hline Jinno et al. ${ }^{(15)}$ & 1 & 31 & 0 & 0 & - & 1 \\
\hline Bristow et al.(6) & 1 & 28 & 0 & 1 & - & - \\
\hline Anderson et al.(2) & 1 & 36 & 0 & 1 & - & 1 \\
\hline Chen $^{(8)}$ & 1 & 38 & 0 & 1 & - & 1 \\
\hline Fishman et al. ${ }^{(10)}$ & 7 & $26 / 34$ & 5 & 2 & - & 4 \\
\hline Ishii et al..$^{(14)}$ & 6 & $39 / 40$ & 4 & 2 & - & 1 \\
\hline Montes et al. ${ }^{(27)}$ & 1 & 28 & 0 & 1 & - & - \\
\hline Abbi et al. ${ }^{(1)}$ & 1 & 37 & 0 & 1 & - & - \\
\hline Amr et al.(3) & 1 & 30 & 0 & 0 & - & - \\
\hline Bruchim et al..$^{(7)}$ & 2 & $26 / 41$ & 0 & 2 & 1 & 1 \\
\hline Matsui et al..(24) & 18 & $35 / 38 / 40$ & 15 & 3 & 5 & 9 \\
\hline Sebire et al. ${ }^{(31)}$ & 77 & - & 57 & 20 & 2 & 15 \\
\hline Makrydimas et al. ${ }^{(21)}$ & 1 & 36 & 0 & 1 & - & - \\
\hline Wax et al. ${ }^{(38)}$ & 1 & 36 & 0 & 1 & - & - \\
\hline Moini; Riazi(26) & 1 & 36 & 0 & 1 & - & - \\
\hline Bovicelli et al. ${ }^{(5)}$ & 1 & 32 & 0 & 0 & - & - \\
\hline Vaisbuch et al. ${ }^{(37)}$ & 2 & $16 / 26$ & 1 & 1 & 2 & - \\
\hline Marcolles et al. ${ }^{(23)}$ & 4 & $14 / 32 / 38$ & 2 & 2 & - & 1 \\
\hline Hyodo et al..(13) & 1 & 28 & 0 & 1 & - & - \\
\hline Lambert et al. ${ }^{(18)}$ & 2 & $23 / 28$ & 0 & 2 & - & 1 \\
\hline Hamanoue et al..$^{(12)}$ & 1 & 33 & 0 & 1 & - & - \\
\hline Klatt et al. ${ }^{(16)}$ & 1 & 31 & 0 & 1 & - & - \\
\hline Niemann et al. ${ }^{(28)}$ & 8 & 28 & 7 & 1 & - & - \\
\hline Piura et al. ${ }^{(30)}$ & 1 & 28 & 0 & 1 & - & - \\
\hline Dolapcioglu et al.(9) & 2 & 29 & 1 & 1 & 1 & - \\
\hline Total & 159 & - & 103 & 56 & 13 & 47 \\
\hline
\end{tabular}

N: número de casos de GGMC; $n$ : número de casos de gestações finalizadas; GGMC: gestação gemelar com feto normal e mola completa; TGP: doença trofoblástica gestacional persistente. 
Concluímos que, na suspeita de gestação gemelar molar com presença de feto vivo, o primeiro passo deve ser a determinação do cariótipo. A seguir, deve-se expor aos pais a possibilidade de a gestação culminar em parto, de acontecerem complicações maternas durante a gestação e de ocorrer TGP.

A decisão de levar a gestação até o termo dependerá da equipe médica e dos pais.

\section{Referências}

1. ABBI, M. et al. Term twin pregnancy with hydatidiform mole and normal fetus. Arch Gynecol Obstet, v. 262, n. 3-4, p. 189-91, 1999.

2. ANDERSON, C. K. et al. Complete hydatidiform mole with a coexisting healthy, viable fetus near term: a case report. J Reprod Med, v. 41, n. 1, p. 55-8, 1996.

3. ARM, M. F. et al. Triplet pregnancy with hydatidiform mole. Int J Gynecol Cancer, v. 10, p. 76-81, 2000.

4. BLOCK, M. F.; MERILL, J. A. Hidatidiform mole with coexisting fetus. Obstet Gynecol, v. 60, n. 1, p. 129-34, 1982.

5. BOVICELLI, L. et al. Prenatal diagnosis of a complete mole coexisting with a dichorionic twin pregnancy: case report. Hum Reprod, v. 19, n. 5, p. 1231-4, 2004.

6. BRISTOW, R. E. et al. Complete hydatidiform mole and surviving coexisting twin. Obstet Gynecol Surv, v. 51, n. 12, p. 705-9, 1996.

7. BRUCHIM, I. et al. Complete hydatidiform mole with a coexisting viable fetus: report to two cases and review of the literature. Gynecol Oncol, v. 77, n. 1, p. 197-202, 2000.

8. CHEN, E. P. Molar pregnancy and living normal fetus coexistent until term: prenatal biochemical and sonographic diagnosis. Hum Reprod, v. 12, n. 4, p. 853-6, 1997.

9. DOLAPCIOGLU, K. et al. Twin pregnancy with a complete hydatidiform mole and co-existent live fetus: two cases reports and review of the literature. Arch Gynecol Obstet, v. 279, n. 3, p. 431-6, 2009.

10. FISHMAN, D. A. et al. Management of twin pregnancies consisting of a complete hydatidiform mole and normal fetus. Obstet Gynecol, v. 91, n. 4, p. 546-50, 1998.

11. GARBIN, O. et al. How to deal with a rare entity: the coexistence of a complete mole and healthy egg in a twin pregnancy? Fetal Diagn Ther, v. 10, n. 5, p. 337-42, 1995.

12. HAMANOUE, $\mathrm{H}$. et al. Complete hydatidiform mole and normal live birth following intracytoplasmic sperm injection. J Hum Genet, v. 51, n. 5, p. 477-9, 2006.

13. HYODO, M. et al. Molar DNA in maternal serum in a case of $46, X Y$ heterozygous complete hydatidiform mole coexisting with a 46, XX twin live fetus. Clin Chem, v. 51, n. 3, p. 676-7, 2005.

14. ISHII, J. et al. Genetic differentiation of complete hydatidiform moles coexisting with normal fetuses by short tandem repeat derived deoxyribonucleic acid polymorphism analysis. Am J Obstet Gynecol, v. 179, n. 3, pt 1, p. 628-34, 1998.

15. JINNO, M. et al. Hydatidiform mole with a surviving coexisting fetus following in-vitro fertilization. Hum Reprod, v. 9, n. 9, p. 1770-2, 1994.
16. KLATT, T. E.; FRANCIOSI, R. A.; CRUIKSHANK, D. P. Normal fetus with a twin presenting as both a complete hydatidiform mole and placenta previa. Obstet Gynecol, v. 107 , n. 2, pt 2, p. 527-30, 2006

17. KOYAMA, S. et al. A case of complete hydatidiform mole with coexistent fetus: conclusive diagnosis of androgenesis of the molar placenta by variation of paternal acrocentric short arms. Am J Perinatol, v. 27, n. 2, p. 143-9, 2010

18. LAMBERT-MESSERLIAN, G. et al. Second-trimester maternal serum markers in twin pregnancy with complete mole: report of 2 cases. Pediatr Dev Pathol, v. 8, n. 2, p. 230-4, 2005.

19. LEE, S. W. et al. Clinical findings of multiple pregnancy with a complete hydatidiform mole and coexisting fetus. J Ultrasound Med, v. 29, n. 2, p. 271-80, 2010.

20. LURIAN, J. R. Gestacional trophoblastic disease I: epidemiology, pathology, clinical presentation and diagnosis of gestacional trophoblastic disease and managment of hydatidiform mole. Am J Gynecol Obstet, p. 1-9, 2010.

21. MAKRYDIMAS, G. et al. Complete hydatidiform mole and normal live birth: a novel case of conWned placental mosaicism. Hum Reprod, v. 17, n. 9, p. 2459-63, 2002.

22. MALHTRA, N. et al. Hydatidiform mole with coexisting live fetus in dichorionic twin gestation. Eur J Obstet Gynecol Reprod Biol, v. 94, n. 2, p. 301-3, 2001.

23. MARCORELLES, P. et al. Diagnosis and outcome of complete hydatidiform mole coexisting with a live twin fetus. Eur J Obstet Gynecol Reprod Biol, v. 118, n. 1, p. 21-7, 2005.

24. MATSUI, H. et al. Hydatidiform mole coexistent with a twin live fetus: a national collaborative study in Japan. Hum Reprod, v. 15, n. 3, p. 608-11, 2000.

25. MILLER, D. et al. Complete hydatidiform mole coexisting with a twin live fetus. Clinical course of four cases with complete cytogenic analysis. Gynecol Oncol, v. 50, n. 1, p. 119-23, 1993.

26. MOINI, A.; RIAZI, K. Molar pregnancy with a coexisting fetus progressing to a viable infant. Int J Gynecol Obstet, v. 82, n. 1, p. 63-4, 2003.

27. MONTES-DE-OCA-VALERO, F.; MACARA, L.; SHAKER, A. Twin pregnancy with a complete mole and coexisting fetus following in-vitro fertilization. Hum Reprod, v. 14, n. 11, p. 2905-7, 1999.

28. NIEMANN, I.; SUNDE, L.; PETERSEN, L. K. Evaluation of the risk of persistent trophoblastic disease after twin pregnancy with diploid hydatidiform mole and coexisting fetus. Am J Obstet Gynecol, v. 197, n. 1, p. 451-5, 2007. 
29. OGURA, T. et al. Complete mole coexistent with a twin fetus. J Obstet Gynaecol, v. 32, n. 6, p. 593-601, 2006.

30. PIURA, B. et al. Twin pregnancy with complete hydatidiform mole and surviving co-existent fetus. Arch Gynecol Obstet, v. 278, n. 4, p. 377-82, 2008.

31. SEBIRE, N. L. et al. Outcome of twin pregnancies with complete hydatidiform mole healthy co-twin. The Lancet, v. 359, n. 9324, p. 2165-6, 2002.

32. SECKL, M. J.; SEBIRE, N. J.; BERKOWITZ, R. S. Gestational trophoblastic disease. Lancet, v. 376, p. 717-29, 2010.

33. SHAHABI, S. et al. Complete hytatidiform mole and coexisting fetus. A case report of two cases with contrasting outcomes. J Reprod Med, v. 42, n. 11, p. 756-60, 1977.

34. STELLER, M. A. et al. Clinical features of multiple conception with parcial or complete molar pregnancy and coexisting fetuses. J Reprod Med, v. 39, n. 3, p. 147-54, 1994.

35. SUGIGAMA, S. et al. Genetically identified complete hydatiform mole coexisting with a live twin fetus: comparison with conventional diagnosis. Gynecol Obstet Invest, v. 64, n. 4, p. 228-31, 2007.

36. SURI, S.; DAVIES, M.; JAUNIAISS, X. Twin pregnangy presenting as a previa complete hydatiform mole and coexintig fetus complicated by a placental abscess. Fetal Diagn Thear, v. 26, p. 181-4, 2009.

37. VAISBUCH, E. et al. Twin pregnancy consisting of a complete hidatidiform mole and co-existent fetus: report of two cases and a review of literature. Gynecol Oncol, v. 98, n. 1, p. 19-23, 2005.

38. WAX, J. R. et al. Prenatal diagnosis by DNA polymorphism analysis of complete mole with coexisting twin. Am J Obstet Gynecol, v. 188, n. 4, p. 1105-6, 2003. 